\title{
SAGE OIL CONTROLS DIET INDUCED OBESITY BY MODULATING OF ADIPONECTIN/AMPK SIGNALING PATHWAY IN RATS \\ BY
}

Omar MM. Mohafez ${ }^{1}$, Hafez R. Madkor ${ }^{1}$, Ahmed A. Abdel-Ghany ${ }^{1}$, Maged E. Mohamed $^{2,3}$

\section{FROM}

${ }^{1}$ Department of Biochemistry, Faculty of Pharmacy, Al-Azhar University, Assiut, Egypt.

${ }^{2}$ Department of Pharmacognosy, College of Clinical Pharmacy, King Faisal University, Alhasa, KSA.

${ }^{3}$ Department of Pharmacognosy, Faculty of Pharmacy, University of Zagazig, Zagazig 44519, Egypt

\begin{abstract}
Obesity represents a major challenge to the pharmaceutical community due to the minimal availability of anti-obesity drugs and drawbacks of current weight-loss agents. The study was designed to evaluate the use of sage oil as a potential anti-obesity agent via its effect on different physiological, biochemical, and hormonal parameters in rats. Rats were divided into two groups: one group was continued on a standard commercial rodent diet and served as the non-obese control. The other group was fed a high-fat diet for seven weeks to prepare an obese rat model. Then, the obese rats were divided into three groups; one group remained without treatment as positive control, the other two groups received $100 \mathrm{mg} / \mathrm{kg}$ orally of the sage oil for 10 or 20 days. The results of the present study showed that treatment with sage oil significantly decreased rats' food intake, epididymal fat, and body weight, and improved their lipid profile levels; it also modulated the leptin resistance. This anti-obesity effect of sage oil was associated with the increased expression of adiponectin and decreased both of leptin and sterol regulatory element-binding protein (SREBP-1c) levels, and this effect may be mediated through AMPK activation. Additionally, the sage oil did not have any effects on the liver's integrity, as was proven by the non-altered ALT levels.
\end{abstract}

Keywords: Obesity; Sage; Adiponectin; AMPK

\section{INTRODUCTION}

Obesity is a complex growing global health problem leading to chronic medical conditions such as cardiovascular disease, metabolic disorders and cancer. Changes in the physiologic function of adipose tissue in obesity include insulin resistance, decreased level of adiponectin, and increased levels of plasminogen activator inhibitor1, leptin and endogenous sex steroids (Bjornvad et al., 2014; Eglit et al., 2013 and Nagaraju et al., 2015). Adipose tissue, in addition to its role as an energy reservoir, modulates energy metabolism via secretion of circulating adipocytokines such as resistin, leptin and adiponectin that appear to be important in regulating insulin 
sensitivity. Obesity, particularly visceral obesity, is associated with insulin resistance (Balsan et al., 2015 and Ceddia et al., 2002).

Adiponectin, known as ACRP30, GBP28, ADIPOQ, and apM1 gene product, is an adipokine hormone that was first discovered in 1995(Scherer $\boldsymbol{e t}$ al., 1995). It is a 244-amino acid polypeptide protein, encoded on chromosome 3q27 (human adiponectin gene, apM1). It has the ability to decrease sugar, lipids, and triglycerides, and plays an important role in metabolic syndrome. One of the main functions of adiponectin is its regulation of insulin sensitivity in our body. It increases the sensitivity of the liver to insulin by reducing the amount of fatty acids taken up by the liver through upregulation of expression of fatty acid transport proteins, which transport triglycerides to the skeletal muscles (Abraham et al., 2017 and Stefan et al., 2002).

Leptin has a molecular mass of $16 \mathrm{kDa}$ and has 167 -amino acids coded by the ob gene and an adipocyte derived hormone that acts as a major regulator for food intake and energy homeostasis and it has an important role in the long-term regulation of body weight (Zhang et al., 2005). Leptin inhibits food intake and increases energy expenditure in animals (Engin, 2017 and Myers et al., 2008). It also increases glucose utilization and fatty acid oxidation (FAO) in certain peripheral tissues including skeletal muscle, without affecting plasma glucose and insulin levels, in rodents (Toda $\boldsymbol{e t}$ al., 2013).

AMP-activated protein kinase (AMPK), a $\alpha \beta \gamma$ heterotrimer, is a regulatory enzyme involved in lipid and energy metabolism (Kahn et al., 2005). Activation of AMPK regulates lipogenesis, fatty acid oxidation and glucose transport (Daval et al., 2006). Phosphorylation of AMPK inhibits lipid biosynthesis by the inactivation of key metabolic enzymes involved in fatty acid and cholesterol synthesis, such as acetyl-CoA carboxylase (ACC) and hydroxy-3-methylglutaryl coenzyme-A reductase (HMGCR) (Kramer et al., 2007 and Rodrigues et al., 2014).

Salvia officinalis (Common sage), a perennial round shrub in the family of Labiatae/Lamiaceae, is native to Mediterranean region and some of the salvia species have been used worldwide as flavoring spices as well as traditional herbal medicine (Jantova et al., 2014 and Martins et al., 2015). Salvia Extract and oil are considered important for drug development, because they are reported to have pharmacological activity in Asia and Middle East. Several studies suggest that sage might potentially provide novel natural treatments for the relief or cure of many serious and life threatening diseases in addition to treating minor common illnesses such as depression, dementia, obesity, diabetes, lupus, heart disease and cancer. This plant is used as medicine in Asia, Africa, Middle East, South America, and some Countries in Europe (Hamidpour et al., 2014). The essential oil of Salvia species can have various compositions depending on the genetic, climates, season and environmental factors (Ghorbani and Esmaeilizadeh, 2017 and Martins et al., 2015). There are some chemical compounds like flavonoids, terpenoids and essential oils present in different species of salvia. Essential oils are very important sources for the screening of anticancer, antimicrobial, antioxidant, and free radical scavenging agents. Salvia officinalis is considered to have the highest amount of essential oil compared to the other species of salvia (Bouajaj et al., 2013; Jug-Dujakovic et al., 2012 and Placha et al., 2015) 
Anti-obesity drugs are important adjunctive therapy, in combination with lifestyle changes, to improve the obesity status of obese individuals. Unfortunately, many of the reported anti-obesity drugs are associated with many limitation due to their high attrition rates, their burden on the liver, and the lack of long-term morbidity and mortality data (Padwal and Majumdar, 2007). In light of the aforementioned data on the obesity pandemic, the development of safe and effective treatment alternatives should be a priority. This study investigated sage oil as an anti-obesity drug, which is in accordance with the line of alternative developments of natural and safe weight-loss agents.

\section{MATERIAL AND METHODS}

\section{Chemicals and reagents}

Sunflower oil was purchased from a local commercial distributor. Tween 20 was purchased from Sigma-Aldrich Co. (St Louis, MO, USA). A leptin rat enzyme-linked immunosorbent assay (ELISA) kit (ab100773) was obtained from Abcam plc (Cambridge, UK). A rat insulin ELISA kit (80-INSRT-E01) was purchased from ALPCO Diagnostics (Salem, NH, USA). AMPK $\alpha 1 / 2$ (SC-25792), $\beta$-actin (SC-130656), goat anti-rabbit immunoglobulin (Ig)G-horseradish peroxidase (HRP) (SC-2030) antibodies and polyvinylidene fluoride (PVDF) membrane (SC-3723) were purchased from Santa Cruz Biotechnology, Inc. (Dallas, TX, USA). Alanine aminotransferase (ALT), blood glucose, total cholesterol, and high-density lipoprotein (HDL)-cholesterol kits were purchased from Human Geselischanft (Wiesbaden, Germany).

\section{Plant:}

The fresh leaves of sage (Salvia officinalis, family Lamiaceae) were collected from local farms in Alahsa, eastern province, Kingdom of Saudi Arabia, in January 2017. The plant was preserved in and mixture of Ethyl alcohol: glycrine: water (1:1:1) and then kindly identified by Dr. A. H. Abdel-Baset, plant taxonomist, Egyptian Agricultural museum, Cairo, Egypt. Voucher specimens (No. LM015) were deposited in the herbarium of college of Clinical Pharmacy, King Faisal University, Saudi Arabia.

\section{Isolation of the essential oils:}

The fresh leaves of sage $(300 \mathrm{~g})$ were hydrodistilled using Clevenger-type apparatus for 3 hours producing yellowish transparent oil with $2.2 \%$ yield. The oil was dried over anhydrous sodium sulphate and kept at $4^{\circ} \mathrm{C}$ until further analyses.

\section{Gas chromatography analysis:}

Gas chromatography/flame ionization (GC/FID) analysis was done using GC2010 Plus, Shimadzu Corporation, Kyoto, Japan, gas chromatograph equipped with FID-2010 Plus detector. The following conditions were applied; column, RTX$5 \mathrm{MS}{ }^{\circledR}$ fused silica capillary $(30 \mathrm{~m} \times 0.25 \mathrm{mmi} . \mathrm{d}$ and $0.25 \mu \mathrm{m}$ film thickness $)$; carrier gas $\mathrm{He}(2 \mathrm{ml} / \mathrm{min})$; temperature $300^{\circ} \mathrm{C}$, injection temperature $250^{\circ} \mathrm{C}$; oven temperature program: initial temperature $45^{\circ} \mathrm{C}, 2 \mathrm{~min}$ isothermal, $300^{\circ} \mathrm{C}, 4^{\circ} \mathrm{C} / 1 \mathrm{~min}$, then $20 \mathrm{~min}$ isothermal; split ratio was 1:15, injection volume is $5 \mu 1$. Gas chromatography/mass spectrometry (GC/MS) data were recorded on GCMS-QP2010 Plus, Shimadzu Corporation, Kyoto, Japan. The ionization energy for the mass spectrometer was $70 \mathrm{eV}$. Split ratio was 1:30; other conditions were identical to those mentioned for GC/FID. 


\section{Components identification:}

Kovat's retention indices (RI) were calculated with respect to a set of co-injected standard hydrocarbons (C10-C28). Compounds were identified by comparing their spectral data and retention indices with Wiley Registry of Mass Spectral Data $10^{\text {th }}$ edition (April 2013), NIST 11 Mass Spectral Library (NIST11/2011/EPA/NIH) and literature data (Adams, 2007). Most of non-identified components are present as traces with relative abundances of less than $0.01 \%$.

\section{Animals:}

Twenty-eight male Sprague-Dawley rats weighing (170 $\pm 25 \mathrm{~g})$ were obtained from the animal house, Assiut University, Egypt. The rats were kept in standard laboratory conditions $\left(23^{\circ} \mathrm{C} \pm 1^{\circ} \mathrm{C}\right)$ and maintained on a standard commercial rodent diet using a 12-hour light/dark cycle for one week as an accommodation period.

\section{Experimental design:}

All animal experimental procedures and protocols were performed in accordance with the Guidelines for the Ethical Conduct for Use of Animals in Research. Rats were divided into two groups after acclimating to the facility: one group $(n=7)$ was continued on a standard commercial rodent diet and served as the non-obese control (NO). The other group $(\mathrm{n}=21)$ was fed a high-fat diet (HFD; a rodent diet with $60 \%$ of its energy provided from fat) for 7 weeks to prepare the obese animal model. After 7 weeks, the obese rat group was divided into three groups: untreated obese rats (obese); obese rats that received $100 \mathrm{mg} / \mathrm{kg}$ of the sage oil diluted in sunflower oil for 10 days (S-10) or 20 days (S-20). All obese rats were fed the HFD during the treatment period, and the diluted oil was administered by oral gavage. By the end of each designated time, the rats were sacrificed and their blood, liver tissues and epididymal fat were collected. The serum was separated by centrifugation of the blood at 3,000 rpm for 10 minutes, and samples were stored at temperatures of $-20^{\circ} \mathrm{C}$ until analysis.

\section{Determination of physiological and biochemical parameters:}

Blood glucose, ALT, total cholesterol, and HDL levels were measured by using colorimetric kits provided by Human Geselischanft (Wiesbaden, Germany) according to manufacturer protocols. Food intake by all rats was determined according to (Bjornvad et al., 2014), where the food intake of the obese group was considered to be $100 \%$, and the food intake of the other groups was calculated every 3 days. The body weight ( $\mathrm{g}$ ) and epididymal fat weight $(\mathrm{g})$ of all groups were also determined as biochemical parameters.

\section{Hormonal determination:}

Serum insulin was measured by using rat insulin ELISA kit (80-INSRT-E01) that was purchased from ALPCO Diagnostics (Salem, NH, USA). Leptin was determined in serum using leptin rat enzyme-linked immunosorbent assay (ELISA) kit (ab100773) that was obtained from Abcam plc (Cambridge, UK). Adiponectin, AMPK and SREP-1c expression were determined in the epididymal fat by Western blot analysis, as will be described. Similarly, AMPK was also determined in the liver homogenate by Western blot analysis.

Western blot analysis was performed as described by (Takasawa $\boldsymbol{e t}$ al., 2008). Briefly, liver or epididymal fat samples were homogenized in radioimmunoprecipitation 
assay (RIPA) buffer with a protease inhibitor; the total protein extracted from those homogenates were quantified and $50 \mu \mathrm{g}$ of the total extracted protein was separated by sodium dodecyl sulfate (SDS)-polyacrylamide gel electrophoresis (PAGE) and blotted onto PVDF membranes. The membranes were blocked by incubation in Tris-buffered saline (TBS) containing 3\% bovine serum albumin and $0.1 \%$ Tween 20 for 1 hour at room temperature. After washing with TBS containing $0.1 \%$ Tween 20 , the membranes were incubated with the primary antibodies (1:300 dilution) for 2 hours at room temperature, and they were then incubated with the secondary antibody (goat anti-rabbit HRP-conjugated at a 1:5000 dilution). In the epididymal fat experiment, loading uniformity was assessed by Ponceau $S$ staining of the membranes after blotting due to the low expression of $\beta$-actin in the adipose tissue.

\section{Statistical analysis:}

Data were expressed as the mean \pm standard deviation (SD) of at least three independent experiments $(n=7)$. Statistically significant differences between the groups were determined by means of one-way analysis of variance (ANOVA) followed by Tukey's multiple comparison test. In all cases, probability values of $P<0.05$ were taken as statistically significant

\section{RESULTS:}

\section{Effect of sage oil on some physiological and biochemical parameters:}

The rats were divided into two main groups; one group $(n=7)$ served as the nonobese control and other group $(n=21)$ was served as obese group. Obese group was fed a HFD for 7 weeks and subdivided into three groups: untreated obese rats (obese); obese rats that received $100 \mathrm{mg} / \mathrm{kg}$ of the sage oil diluted in sunflower oil for 10 days (S-10) or 20 days (S-20). The oil was dispensed orally to the rats, and all results were taken in comparison with the untreated obese group (obese) unless otherwise specified. To test the effect of sage oil on liver integrity, the ALT level, which is one of the most important liver integrity enzymes, was evaluated. Administering the sage oil did not significantly alter the ALT levels at any time point (Table 1). The effect of sage oil on fasting blood glucose and insulin levels was not significantly affected. Feeding rats with a HFD for 7 weeks significantly $(P<0.001)$ elevated their total cholesterol levels and decreased their HDL levels when compared with the non-obese control group (Table-1). 
Table-1. Physiological and biochemical parameters for the different groups and time points: none-obese (NO) obese untreated (obese), (S-10), and (S-20), after 10 and 20 days from treatment with sage oil.

\begin{tabular}{|c|c|c|c|c|}
\hline Parameters & NO & Obese & S-10 & S-20 \\
\hline $\operatorname{ALT}(\mathbf{U} / \mathbf{L})$ & $45.25 \pm 4.11$ & $47.25 \pm 3.3$ & $46 \pm 2.71$ & $47.5 \pm 4.8$ \\
\hline $\begin{array}{l}\text { Fasting blood glucose } \\
(\mathbf{m g} / \mathrm{dl})\end{array}$ & $86.75 \pm 2.75$ & $89.5 \pm 6.45$ & $83.25 \pm 3.1$ & $81.5 \pm 4.65$ \\
\hline Insulin (ng/ml) & $1.39 \pm 0.09$ & $1.5 \pm 0.07$ & $1.34 \pm 0.11$ & $1.29 \pm 0.1$ \\
\hline Total cholesterol $(\mathrm{mmol} / \mathrm{L})$ & $2.77 \pm 0.12^{* * *}$ & $3.68 \pm 0.09$ & $3.52 \pm 0.15$ & $\begin{array}{l}3.20 \\
0.11^{*}\end{array}$ \\
\hline HDL-cholesterol (mmol/L) & $1.52 \pm 0.05^{* * *}$ & $1.19 \pm 0.07$ & $1.22 \pm 0.03$ & $1.37 \pm 0.04 *$ \\
\hline Epididymal fat (gm) & $1.9 \pm 0.15^{* * *}$ & $5.66 \pm 0.86$ & $5.32 \pm 0.66$ & $3.92 \pm 0.65^{*}$ \\
\hline Body weight (gm) & $\begin{array}{l}219 \\
12.67 * * *\end{array}$ & $\begin{array}{l}284.5 \\
17.15\end{array}$ & $\pm \begin{array}{l}274 \\
15.07\end{array}$ & $247 \pm 10.8^{*}$ \\
\hline Food intake (gm) & $101 \pm 2.64$ & $100 \pm 1.00$ & $93.3 \pm 3.51$ & $\begin{array}{l}81.33 \\
3.2^{*}\end{array}$ \\
\hline
\end{tabular}

Data are represented as means $\pm \mathrm{SD}(\mathrm{n}=5) .{ }^{*} \mathrm{P}<0.05$ and $* * * \mathrm{P}<0.001$ when compared with obese untreated values.

Treatment with sage oil for 20 days was showed a significant $(P<0.05)$ reduction in total cholesterol levels in obese rats. Additionally, sage oil significantly $(P<0.05)$ increased the level of HDL-cholesterol after 20 days however no significant alterations were observed after 10 days of the oil administration. Table- 1 indicates that there was a significant $(P<0.001)$ increase in epididymal fat weight following administration of the HFD in comparison to the non-obese control group. This fat accumulation is significantly $(P<0.05)$ reduced following the administration of the sage oil for 20 days. The HFD induced a significant $(P<0.001)$ increase in the rats' body weight when compared to the non-obese rats, although the amount of food intake in both groups was similar with no significant difference. After sage oil administration, no significant difference was detected in the rats' body weight after 10 days but after 20 days a significant $(P<0.05)$ reduction in the rats' body weight was found.

\section{Effect of sage oil on rats' hormone profile:}

The HFD induced a significant $(P<0.001)$ increase in the leptin level and administration of sage oil for 10days decreased the leptin level insignificantly in comparison to the obese group but after 20days of treatment a significant reduction was noted in the leptin level (Figure-1). 


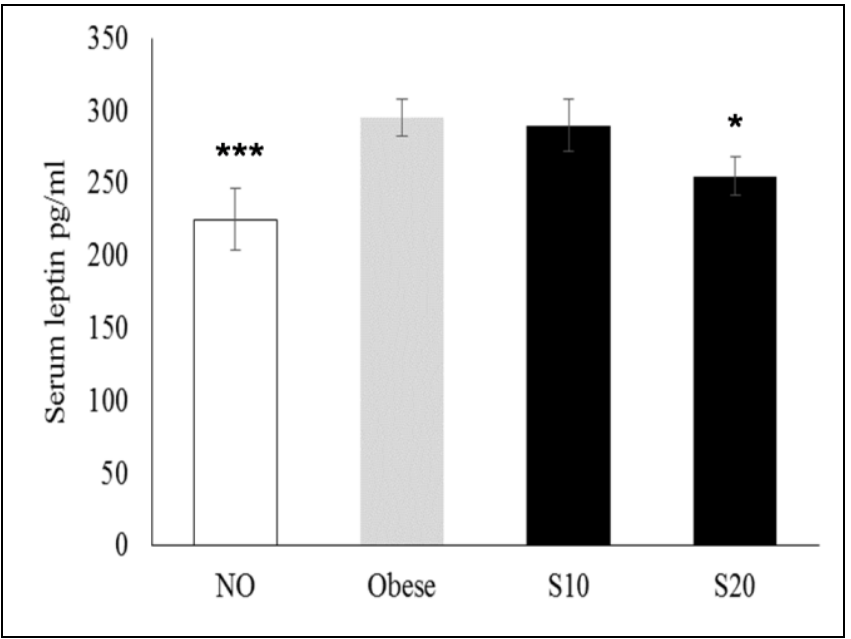

Figure-1: Effect of sage oil on leptin expression: Serum level of leptin determined by ELISA. NO: non-obese group, Obese: obese untreated group, S-10 and S-20: sage oil treated groups after 10 and 20 days. Data are represented as means $\pm \mathrm{SD},(n=7)$. * $\mathrm{P}<$ 0.05 compared with obese untreated values.

On the other hand, a significant $(P<0.01)$ decrease in adiponectin levels in the epididymal fat can be observed following the induction of obesity. The low adiponectin level not only reverted back to the normal level but it was also significantly $(P<0.05$, $P<0.001)$ elevated and exceeded the normal reading as well; following the administration of sage oil for 10 and 20 days respectively (Figure 2).

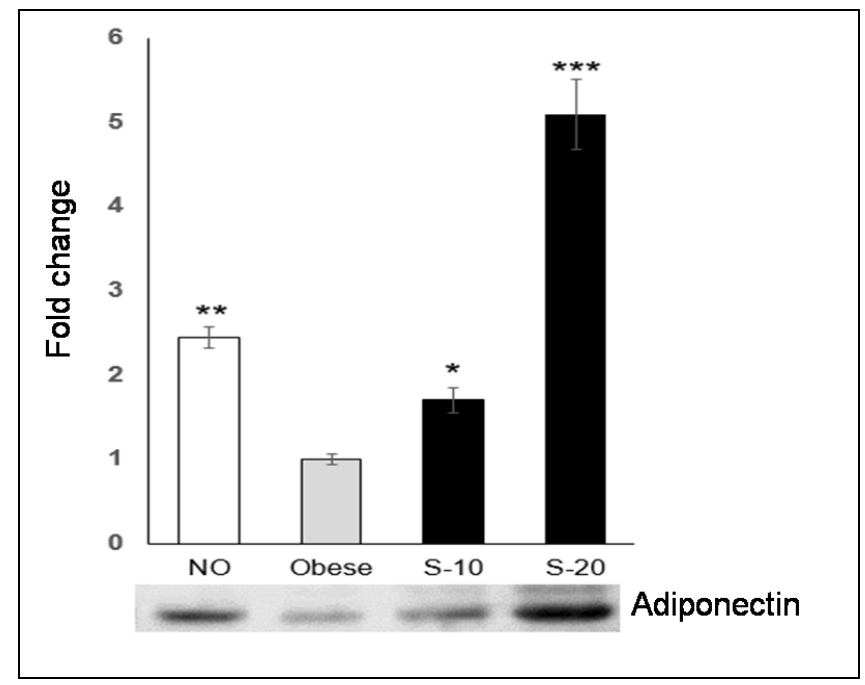

Figure 2. Effect of sage oil on adiponectin expression: Adiponectin expression was determined by Western blot analysis in the epididymal fat. Loading uniformity was assessed by Ponceau $S$ staining of the membranes after blotting because of low expression of $\beta$-actin in the adipose tissue. Data are represented as means $\pm S D, n=7$; $* \mathrm{P}<0.05$, and $* * * \mathrm{P}<0.001$ when compared with obese untreated values. 
HFD resulted in a significant overexpression of sterol regulatory element-binding protein (SREBP-1c) with $P<0.01$ as shown in (Figure-3) and administration of sage oil for 20 days almost normalized the expression of SREBP-1c level $(P<0.01)$.

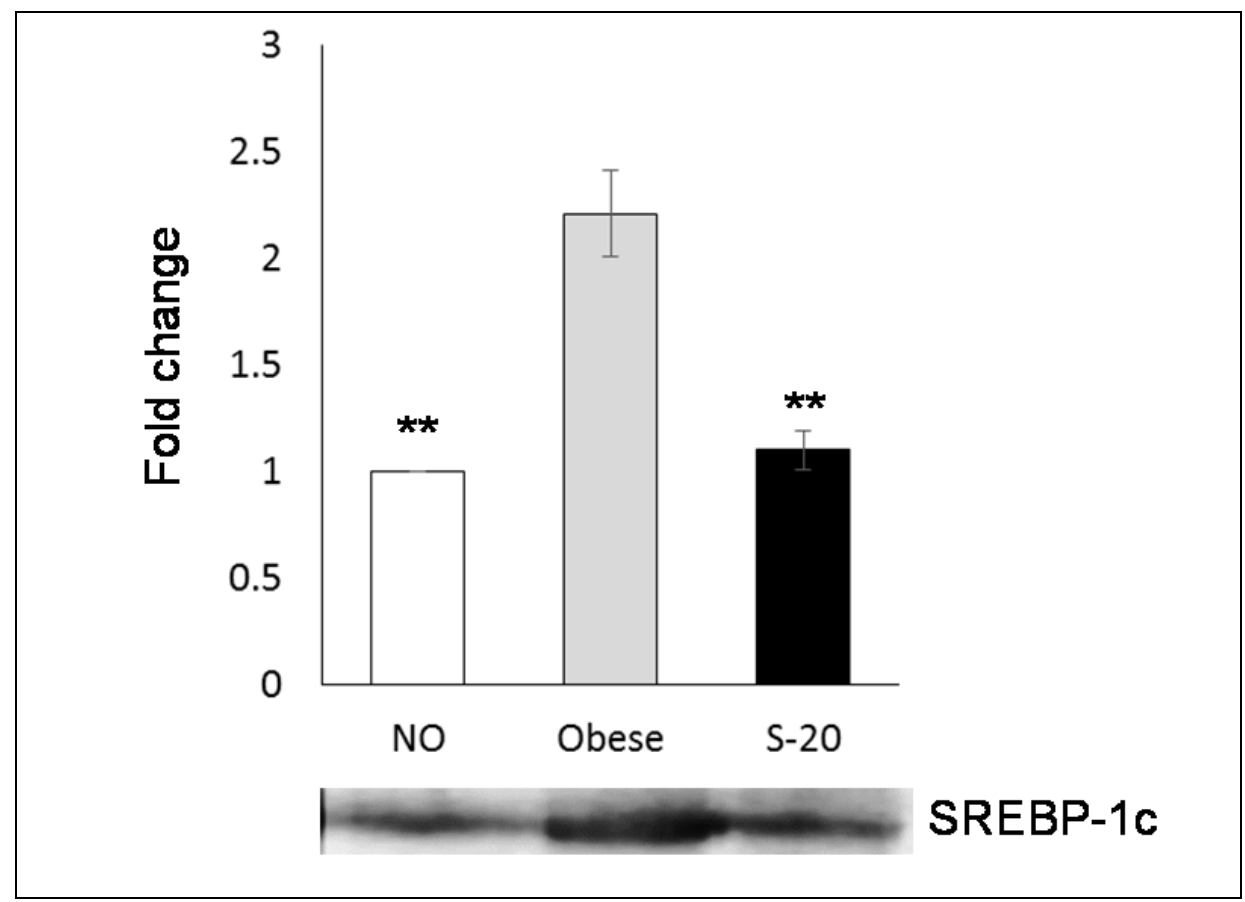

Figure 3. Effect of sage oil on SREBP-1c expression: SREBP-1c expression is determined by Western blot analysis in the epididymal fat. Loading uniformity was assessed by Ponceau $S$ staining of the membranes after blotting because of low expression of $\beta$-actin in the adipose tissue. Data are represented as means $\pm S D, n=5$; $* * \mathrm{P}<0.01$ when compared with obese untreated values.

Western blot analysis was performed on both liver and epididymal fat homogenates to investigate the effect of sage oil on AMPK expression. The analysis of epididymal fat AMPK levels revealed a sharp and significant $(P<0.001)$ increase in the hormone's expression in both S10 and S20 groups, which was nearly 1.9 and 5.4 folds respectively following the administration of the sage oil (Figure 4A). Similarly, a significant overexpression of AMPK was detected in the liver homogenate following oil administration with $P<0.001$ for both S10 and S-20 groups (Figure 4B). 


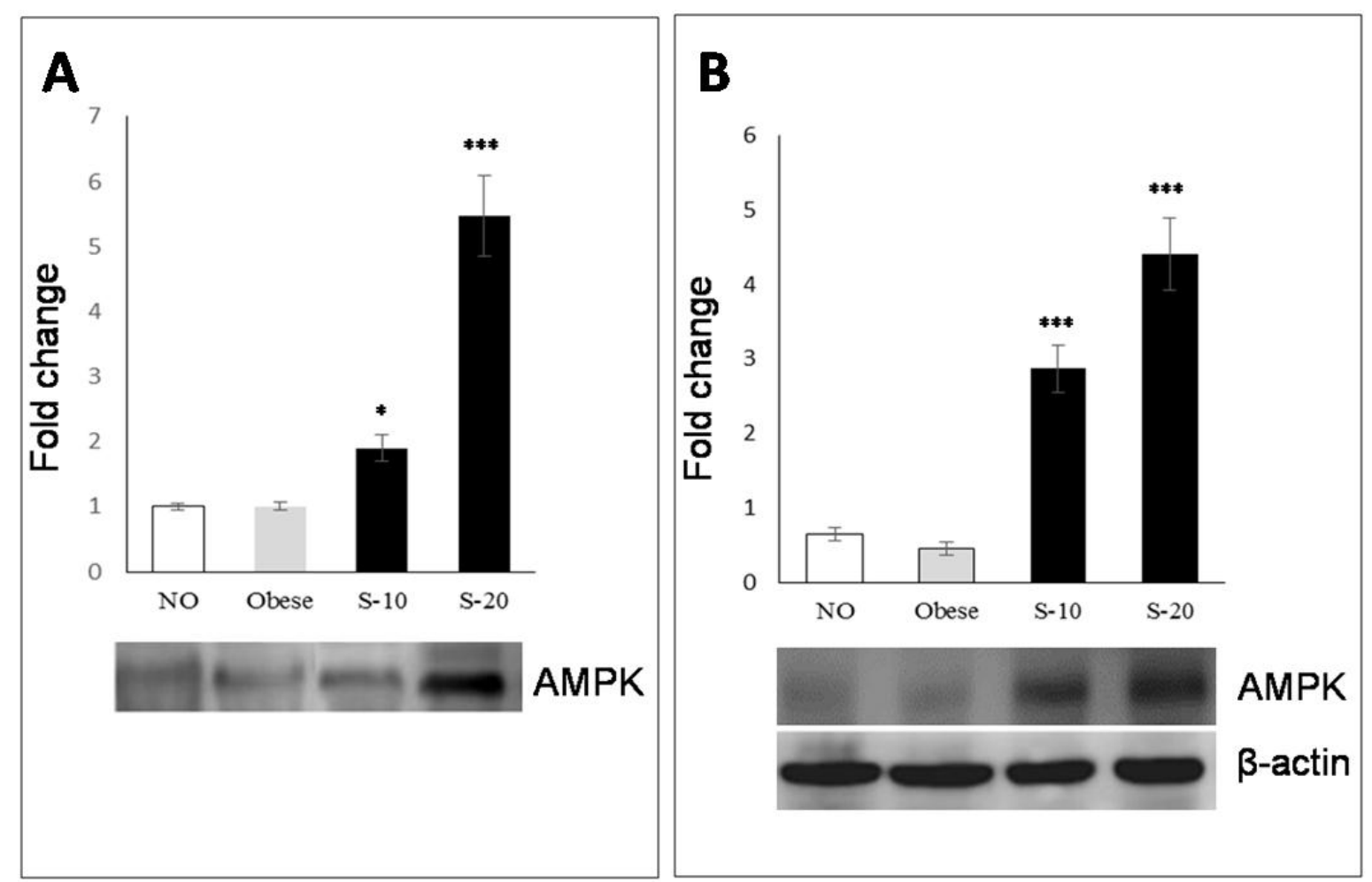

Figure 4. Effect of sage oil on AMPK expression: (A) Expression of AMPK in adipose tissue homogenate. The expression was determined by Western blot analysis in the epididymal fat. Loading uniformity was assessed by Ponceau $S$ staining of the membranes after blotting because of low expression of $\beta$-actin in the adipose tissue. (B) Expression of AMPK in liver homogenate. Data are represented as means $\pm S D, n=5$; $* \mathrm{P}<0.05, * * \mathrm{P}<0.01$ and $* * * \mathrm{P}<0.001$ when compared with obese untreated values.

\section{DISCUSSION:}

Obesity is characterized by dyslipidemia and the accumulation of fat in the abdominal region. Table-1 illustrates that there was a reduction in the rats' body weight and epididymal fat weight in sage oil treated groups. This reduction is associated with reduced food intake, and it may be influenced by the rats' decreased appetite for food intake and this could be attributed to increased levels of lipolysis by the administration of sage oil. Sage oil significantly reduced the total cholesterol levels and increased the HDL levels in a time-dependent manner. This may be attributed to gene expression regulation, particularly among those genes involved in lipid metabolism. This modulatory effects on lipid profile and body weight may be attributed to activation of PPAR-gamma which is a regulator of some genes that involved in energy spending as well as lipid and glucose metabolism and its activation improves the HDL/LDL ratio and reduce the size of adipose tissue (Christensen $\boldsymbol{e t}$ al., 2010). Also, these modulatory effects may be due to negative feedback effects of sage oil on food intake that observed in our study where decreased by $20 \%$ in the obese treated rats after 20 days. In addition, the reduction of body weight and obesity may be due to an inhibitory effect of sage oil on pancreatic lipase activity, Some investigators using the complete sage leave extract revealed that this effect is attributed to carnosic acid and creasol in the extract (Ninomiya et al., 2004) and this hypothesis supports our results where we use sage oil which contain higher concentration of these compounds than the complete extract. HFD 
induced over expression of (SREBP)-1c. The obtained results were in agreement with (Shimano, 2015) who reported that an excess energy state activates (SREBP)-1c, a master transcription factor for fatty acid synthesis causing the accumulation of lipids leading to fatty liver, insulin resistance, insulin secretion defects, and dyslipidemia. Sage oil normalized the (SREBP)-1c levels after 20days of treatment.

Leptin, a pleiotropic hormone, plays a role in appetite suppression and it increases energy expenditure (Placha et al., 2015). It acts as a signal of the body's nutritional status to the reproductive, immune, cardiovascular, skeletal, and thyroid systems. Moreover, it plays a regulatory role in blood sugar levels through its effects on the liver, pancreas, and blood pressure, both directly and via the sympathetic nervous system (Placha $\boldsymbol{e t}$ al., 2015). Leptin and adiponectin are considered the major players in obesity metabolism, as they primarily regulate adenosine monophosphate (AMP)activated protein kinase (AMPK) activity (Dzamko and Steinberg, 2009). Leptin activates many kinases and signal transducers, which inhibit orexigenic peptides and activate anorexigenic peptides (Ahima, 2006). Leptin restricts food intake and suppresses hypothalamic AMPK activity, while adiponectin stimulates hypothalamic AMPK, which controls energy expenditure (Stark et al., 2013). Furthermore, leptin decreases glucose, recovers insulin resistance, diminishes hyperlipidemia (Havel, 2002), and stimulates lipolysis through central and peripheral pathways involving AMPK (Ahima, 2006). AMPK switches off pathways of adenosine triphosphate (ATP) consumption and switches on the ATP production pathways, such as fatty acid oxidation and glucose uptake. Moderated AMPK activity in the liver, muscle, adipose tissue, and other peripheral tissues is a predisposing factor for obesity and diabetes as it reduces one's exercise capacity and glucose tolerance (Stark et al., 2013). Sage oil relieved the leptin resistance and this was pronounced by significant leptin reduction after 20days of treatment. The leptin has a direct effect on the hypothalamus, which reduces one's appetite and subsequently decreases food intake; this may explain the reduction in body weight observed in the present study. Also sage oil enhanced the over expression of adiponectin. Both of leptin and adiponectin are the main players in AMPK activation which controls energy expenditure.

Activated AMPK, through high levels of adiponectin, stimulates fatty acid oxidation, mitochondrial biogenesis, and glycolysis, and it also inhibits glycogen, fatty acid, and protein synthesis and gluconeogenesis; it also has a direct appetite-regulating effect in the hypothalamus ( $\mathbf{L i m}$ et al., 2010). The results of this study indicated that sage oil increased the expression of adiponectin, and this was concomitant with the over-expression of AMPK in both the epididymal fat and liver, implying that sage oil activity is associated with the AMPK pathway.

In the presented study, sage oil showed an anti-obesity effect when administered to the HFD-induced obese rats. The oil was able to ameliorate the alterations in the lipid profile caused by the HFD, and it improved both insulin and blood sugar levels as an indication of reduced insulin resistance. Notably, sage oil reduced both the epididymal fat weight and food intake, which are considered challenges for obese patients. The use of sage oil was found to be safe, as it did not place any burden on the liver; this is in contrast to many other anti-obesity drugs, which are all associated with hepatic problems. Sage oil enhanced leptin and adiponectin levels in both the serum and epididymal fat, and it also activated AMPK in both the epididymal fat and liver. 
The authors of this study recommend the use of sage oil as a potential antiobesity agent against diet-induced obesity, which can enhance energy expenditure and reduce appetite, epididymal fat, and body weight without affecting the liver. This recommendation requires further investigations using clinical models to confirm the medicinal properties of this preparation on humans.

\section{REFERENCES:}

Abraham PA, Attipoe S, Kazman JB, Zeno SA, Poth M, Deuster PA. (2017): Role of plasma adiponectin /C-reactive protein ratio in obesity and type 2 diabetes among African Americans. Afr Health Sci; 17: 99-107.

Adams R.P., (2007): Identification of essential oil components by gas chromatography/mas spectrometry. $4^{\text {th }}$ eddittion edn. Allured puplishing corporation, Chicago, lllinois, USA.

Ahima RS. (2006): Adipose tissue as an endocrine organ. Obesity (Silver Spring); 14 Suppl 5: 242s-249s.

Balsan GA, Vieira JL, Oliveira AM, Portal VL. (2015): Relationship between adiponectin, obesity and insulin resistance. Rev Assoc Med Bras (1992); 61: 7280.

Bjornvad CR, Rand JS, Tan HY, Jensen KS, Rose FJ, Armstrong PJ, Whitehead JP. (2014): Obesity and sex influence insulin resistance and total and multimer adiponectin levels in adult neutered domestic shorthair client-owned cats. Domest Anim Endocrinol; 47: 55-64.

Bouajaj S, Benyamna A, Bouamama H, Romane A, Falconieri D, Piras A, Marongiu B. (2013): Antibacterial, allelopathic and antioxidant activities of essential oil of Salvia officinalis L. growing wild in the Atlas Mountains of Morocco. Nat Prod Res; 27: 1673-1676.

Ceddia RB, Koistinen HA, Zierath JR, Sweeney G. (2002): Analysis of paradoxical observations on the association between leptin and insulin resistance. FASEB J; 16: $1163-1176$.

Christensen KB, Jorgensen M, Kotowska D, Petersen RK, Kristiansen K, Christensen LP. (2010): Activation of the nuclear receptor PPARgamma by metabolites isolated from sage (Salvia officinalis L.). J Ethnopharmacol; 132: 127-133.

Daval M, Foufelle F, Ferre P. (2006): Functions of AMP-activated protein kinase in adipose tissue. J Physiol; 574: 55-62.

Dzamko NL, Steinberg GR. (2009): AMPK-dependent hormonal regulation of wholebody energy metabolism. Acta Physiol (Oxf); 196: 115-127.

Eglit T, Ringmets I, Lember M. (2013): Obesity, high-molecular-weight (HMW) adiponectin, and metabolic risk factors: prevalence and gender-specific associations in Estonia. PLoS One; 8: e73273.

Engin A. (2017): Diet-Induced Obesity and the Mechanism of Leptin Resistance. $A d v$ Exp Med Biol; 960: 381-397. 
Ghorbani A, Esmaeilizadeh M. (2017): Pharmacological properties of Salvia officinalis and its components. J Tradit Complement Med; 7: 433-440.

Hamidpour M, Hamidpour R, Hamidpour S, Shahlari M. (2014): Chemistry, Pharmacology, and Medicinal Property of Sage (Salvia) to Prevent and Cure Illnesses such as Obesity, Diabetes, Depression, Dementia, Lupus, Autism, Heart Disease, and Cancer. J Tradit Complement Med; 4: 82-88.

Havel PJ. (2002): Control of energy homeostasis and insulin action by adipocyte hormones: leptin, acylation stimulating protein, and adiponectin. Curr Opin Lipidol; 13: 51-59.

Jantova S, Hudec R, Sekretar S, Kucerak J, Melusova M. (2014): Salvia officinalis L. extract and its new food antioxidant formulations induce apoptosis through mitochondrial/caspase pathway in leukemia L1210 cells. Interdiscip Toxicol; 7: 146-153.

Jug-Dujakovic M, Ristic M, Pljevljakusic D, Dajic-Stevanovic Z, Liber Z, Hancevic K, Radic T, Satovic Z. (2012): High diversity of indigenous populations of dalmatian sage (Salvia officinalis L.) in essential-oil composition. Chem Biodivers; 9: 2309-2323.

Kahn BB, Alquier T, Carling D, Hardie DG. (2005): AMP-activated protein kinase: ancient energy gauge provides clues to modern understanding of metabolism. Cell Metab; 1: 15-25.

Kramer DK, Al-Khalili L, Guigas B, Leng Y, Garcia-Roves PM, Krook A. (2007): Role of AMP kinase and PPARdelta in the regulation of lipid and glucose metabolism in human skeletal muscle. J Biol Chem; 282: 19313-19320.

Lim CT, Kola B, Korbonits M. (2010): AMPK as a mediator of hormonal signalling. $J$ Mol Endocrinol; 44: 87-97.

Martins N, Barros L, Santos-Buelga C, Henriques M, Silva S, Ferreira IC. (2015): Evaluation of bioactive properties and phenolic compounds in different extracts prepared from Salvia officinalis L. Food Chem; 170: 378-385.

Myers MG, Cowley MA, Munzberg H. (2008): Mechanisms of leptin action and leptin resistance. Annu Rev Physiol; 70: 537-556.

Nagaraju GP, Aliya S, Alese OB. (2015): Role of adiponectin in obesity related gastrointestinal carcinogenesis. Cytokine Growth Factor Rev; 26: 83-93.

Ninomiya K, Matsuda H, Shimoda H, Nishida N, Kasajima N, Yoshino T, Morikawa T, Yoshikawa M. (2004): Carnosic acid, a new class of lipid absorption inhibitor from sage. Bioorg Med Chem Lett; 14: 1943-1946.

Padwal RS, Majumdar SR. (2007): Drug treatments for obesity: orlistat, sibutramine, and rimonabant. Lancet; 369: 71-77.

Placha I, Ryzner M, Cobanova K, Faixova Z, Faix S. (2015): Effects of dietary supplementation with sage (Salvia officinalis L.) essential oil on antioxidant status and duodenal wall integrity of laying strain growers. Pol J Vet Sci; 18: 741-749. 
Rodrigues SC, Pantaleao LC, Nogueira TC, Gomes PR, Albuquerque GG, Nachbar RT, Torres-Leal FL, Caperuto LC, Lellis-Santos C, Anhe GF, Bordin S. (2014): Selective regulation of hepatic lipid metabolism by the AMPactivated protein kinase pathway in late-pregnant rats. Am J Physiol Regul Integr Comp Physiol; 307: R1146-1156.

Scherer PE, Williams S, Fogliano M, Baldini G, Lodish HF. (1995): A novel serum protein similar to C1q, produced exclusively in adipocytes. J Biol Chem; 270: 26746-26749.

Shimano H. (2015): [SREBP-1c and Elovl6 as Targets for Obesity-related Disorders]. Yakugaku Zasshi; 135: 1003-1009.

Stark R, Ashley SE, Andrews ZB. (2013): AMPK and the neuroendocrine regulation of appetite and energy expenditure. Mol Cell Endocrinol; 366: 215-223.

Stefan N, Vozarova B, Funahashi T, Matsuzawa Y, Weyer C, Lindsay RS, Youngren JF, Havel PJ, Pratley RE, Bogardus C, Tataranni PA. (2002): Plasma adiponectin concentration is associated with skeletal muscle insulin receptor tyrosine phosphorylation, and low plasma concentration precedes a decrease in whole-body insulin sensitivity in humans. Diabetes; 51: 1884-1888.

Takasawa A, Kato I, Takasawa K, Ishii Y, Yoshida T, Shehata MH, Kawaguchi H, Mohafez OM, Sasahara M, Hiraga K. (2008): Mutation-, aging-, and gene dosage-dependent accumulation of neuroserpin (G392E) in endoplasmic reticula and lysosomes of neurons in transgenic mice. J Biol Chem; 283: 35606-35613.

Toda C, Shiuchi T, Kageyama H, Okamoto S, Coutinho EA, Sato T, OkamatsuOgura Y, Yokota S, Takagi K, Tang L, Saito K, Shioda S, Minokoshi Y. (2013): Extracellular signal-regulated kinase in the ventromedial hypothalamus mediates leptin-induced glucose uptake in red-type skeletal muscle. Diabetes; 62: 2295-2307.

Zhang F, Chen Y, Heiman M, Dimarchi R. (2005): Leptin: structure, function and biology. Vitam Horm; 71: 345-372. 


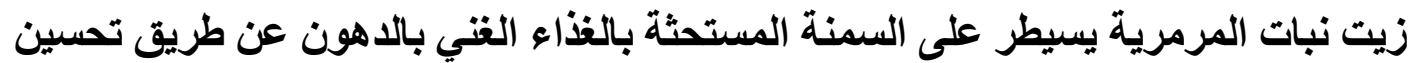

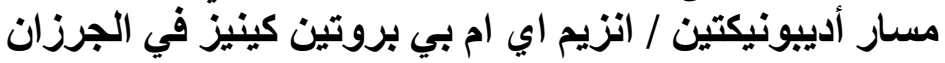

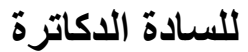

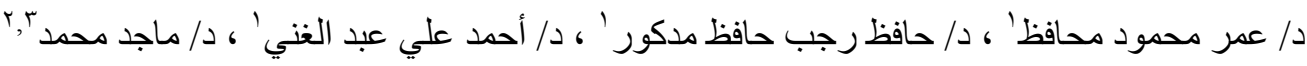

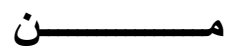

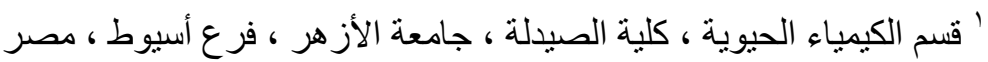

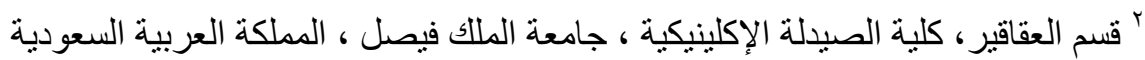

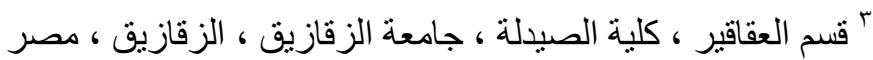

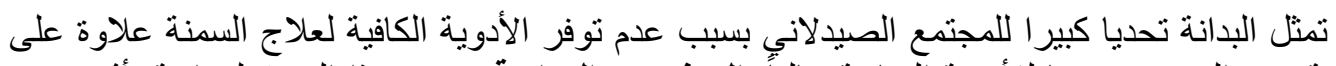

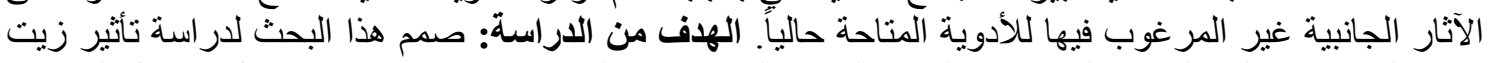

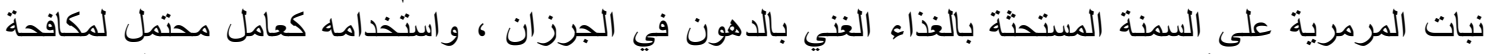

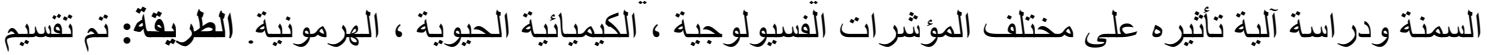

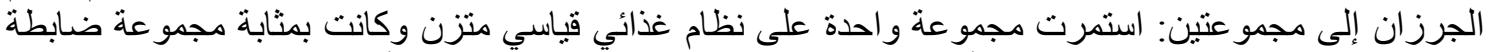

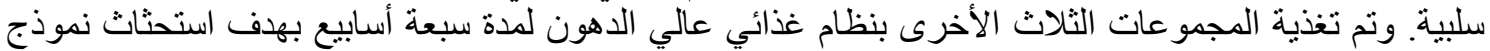

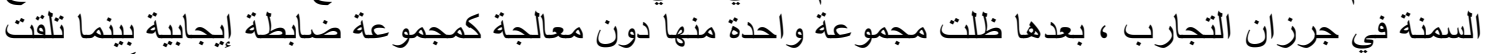

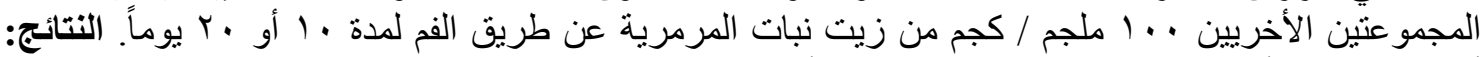

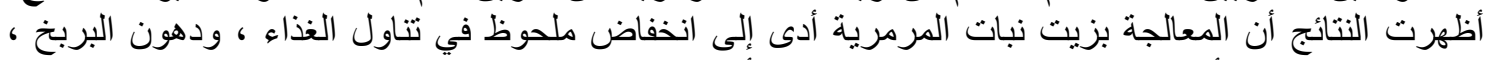

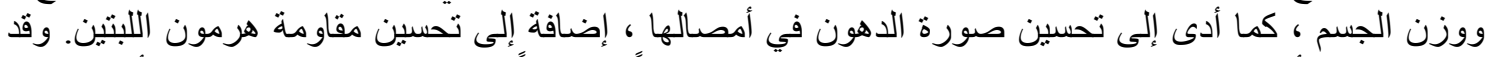

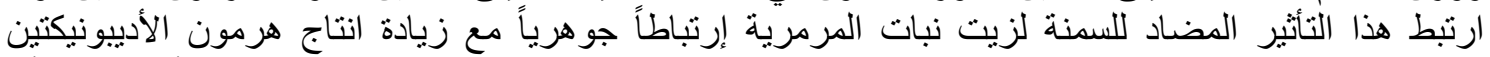

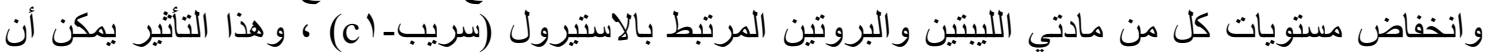

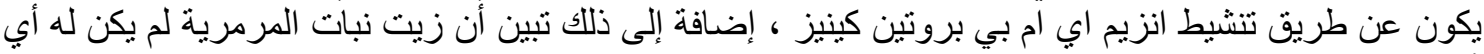

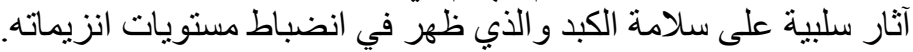

